\title{
Real-space Imaging of Plasmonic Modes of Gold Tapers by EFTEM and EELS
}

Wilfried Sigle ${ }^{1}$, Nahid Talebi ${ }^{1}$, Surong Guo ${ }^{1}$, Martin Esmann ${ }^{2}$, Simon F. Becker $^{2}$, Ralf Vogelgesang ${ }^{2}$, Christoph T. Lienau ${ }^{2}$, Peter A. van Aken ${ }^{1}$

1. Max Planck Institute for Intelligent Systems, Stuttgart, Germany

2. Carl von Ossietzky Universität Oldenburg, Oldenburg, Germany

Plasmonic nanoparticles have been extensively studied in the literature due to their ability of supporting localized surface plasmon (LSP) modes. Such structures can localize optical energy on the nanometer scale which opens up the field of optical nanoantennas. Application of nanoantennas in ultrafast optics requires large bandwidth. Unfortunately, the bandwidths of presently realized nanoantennas are small, despite their large radiative and Ohmic losses. Moreover, the coupling efficiency of far-field optical radiation to single nanoantennas is quite low, which is because of the extremely small volume of interaction.

It was shown theoretically that tapered metallic nanostructures are able to couple propagating surface plasmon polaritons along their shaft adiabatically to nanolocalized plasmons at their apex [1]. This allows such tapered metallic waveguides to being applied in the field of sub-diffraction-limit nanofocusing, ultrafast photoemission, and near-field optical microscopy [2].

Here, we investigated both energy and spatial distribution of plasmonic modes being excited at the tip of gold tapers using electron energy-loss spectroscopy (EELS) and energy-filtering transmission electron microscopy (EFTEM). The EELS signal near the apex shows a broadband local density of optical states, as required for nanoantennas (Fig.1) in contrast to the narrow peaks from plasmon excitations along the shaft. This is also visible from EFTEM images (Fig.2) revealing strong intensity at the apex in the wide energy-loss range from 1.2 to $2.0 \mathrm{eV}$. We discuss the coupling of localized plasmons at the apex to the propagating plasmons at the shaft by considering the coupling efficiency and adiabatic behavior of the taper [4].

\section{References:}

[1] M. Stockman, Phys. Rev. Lett. 93 (2004), p.137404.

[2] M. Esmann et al, Beilstein J Nanotech. 4 (2013), p.603.

[3] N. Talebi et al, Appl. Phys. a-Mater. 116 (2014), p.947.

[4] The research leading to these results has received funding from the European Union Seventh Framework Program [FP/2007-2013] under grant agreement no 312483 (ESTEEM2). 


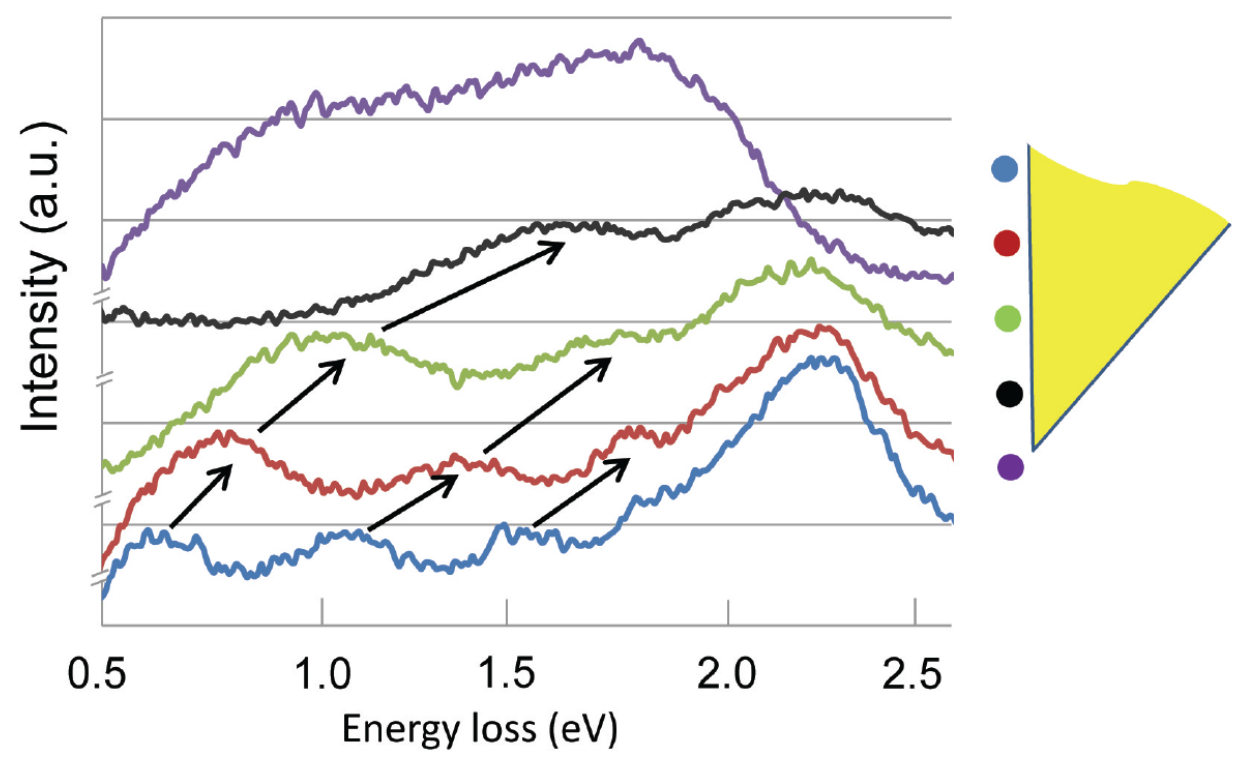

Figure 1. EELS spectra of a gold taper with an opening angle of $\alpha=45^{\circ}$ for electron impact at the apex (purple) and at distances of $275 \mathrm{~nm}$ (black), $504 \mathrm{~nm}$ (green), $733 \mathrm{~nm}$ (red), and $962 \mathrm{~nm}$ (blue) from the apex. The spectra are shifted vertically for clarity. The zero-loss-peak contribution was subtracted from the individual spectra by using a power-law fit.

(a)

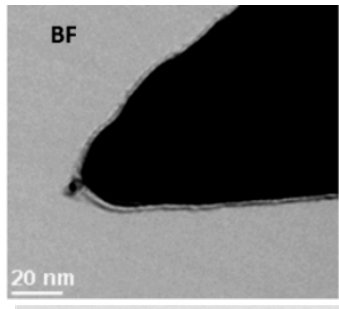

(b)

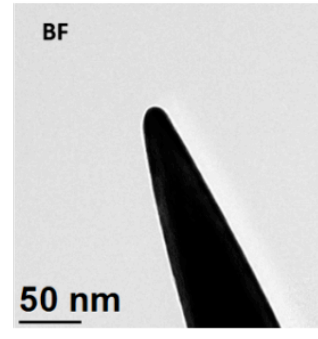

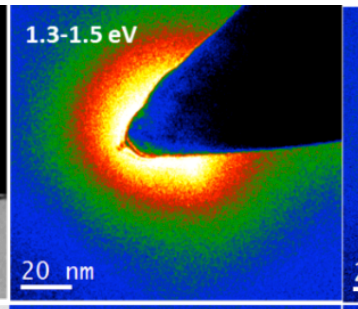
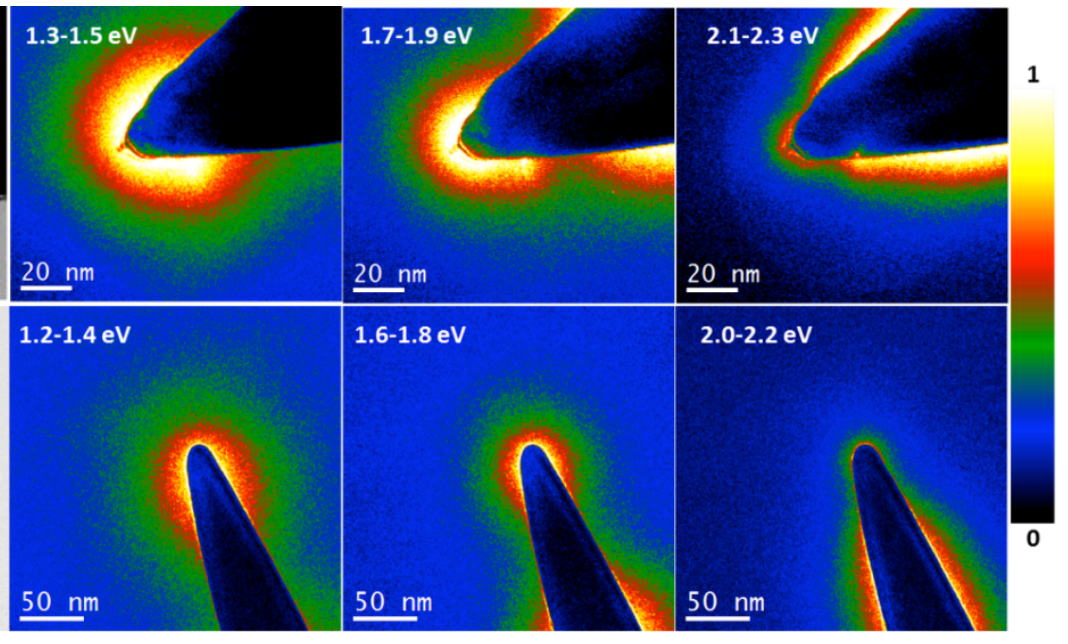

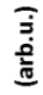

Figure 2. Bright-field image and EFTEM images of conical Au tapers with an opening angle of (a) $35^{\circ}$ in the energy-loss interval from 1.3 to $2.3 \mathrm{eV}$ and of (b) $15^{\circ}$ in the energy-loss interval from 1.2 to $2.2 \mathrm{eV}$. The color bar on the right symbolizes the energy-loss probability which is a measure of the $z$-component of the excited electric field [3]. 